\title{
Correction to: Cloud Computing - CLOUD 2019
}

Dilma Da Silva, Qingyang Wang (1), and Liang-Jie Zhang ()

\section{Correction to: \\ D. Da Silva et al. (Eds.): Cloud Computing - CLOUD 2019, LNCS 11513, https://doi.org/10.1007/978-3-030-23502-4}

The original version of the book was revised; the following corrections have been incorporated:

In Chapter "An Approach to Failure Prediction in Cluster by Self-Updating Cause-and-Effect Graph":

- Typographical errors throughout the chapter were corrected

- Figure 10 was replaced because it was a duplicate
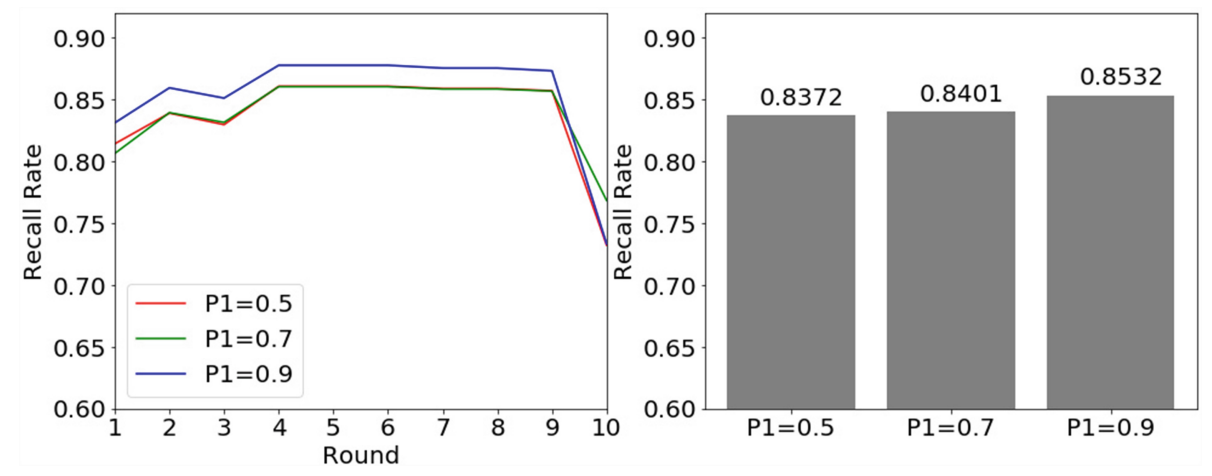

- Unused references were removed

- A missing reference was added

In Chapter "Towards Decentralized Deep Learning with Differential Privacy", typographical errors were corrected to help the reader to better understand the content.

The updated version of these chapters can be found at https://doi.org/10.1007/978-3-030-23502-4_9

https://doi.org/10.1007/978-3-030-23502-4_10

https://doi.org/10.1007/978-3-030-23502-4

(C) Springer Nature Switzerland AG 2019

D. Da Silva et al. (Eds.): CLOUD 2019, LNCS 11513, p. C1, 2019.

https://doi.org/10.1007/978-3-030-23502-4_25 